\title{
Inferring learning from big data: The importance of a transdisciplinary and multidimensional approach
}

\author{
Jason M. Lodge \\ Melbourne Centre for the Study of Higher Education, University of Melbourne, \\ Australia \\ Email: jason.lodge@unimelb.edu.au
}

Sakinah S. J. Alhadad

Learning Futures, Griffith University, Australia

Melinda J. Lewis

Deputy Vice-Chancellor (Education) Portfolio, University of Sydney, Australia

Dragan Gašević

School of Education and Informatics, University of Edinburgh, Scotland, UK

\begin{abstract}
The use of big data in higher education has evolved rapidly with a focus on the practical application of new tools and methods for supporting learning. In this paper, we depart from the core emphasis on application and delve into a mostly neglected aspect of the big data conversation in higher education. Drawing on developments in cognate disciplines, we analyse the inherent difficulties in inferring the complex phenomenon that is learning from big datasets. This forms the basis of a discussion about the possibilities for systematic collaboration across different paradigms and disciplinary backgrounds in interpreting big data for enhancing learning. The aim of this paper is to provide the foundation for a research agenda, where differing conceptualisations of learning become a strength in interpreting patterns in big datasets, rather than a point of contention.
\end{abstract}

Keywords: Learning analytics, inference, predictive modelling, transdisciplinarity

\section{Acknowledgements}

This work was supported by the Australian Research Council (JML) [grant number: SR120300015]. 


\title{
Inferring learning from big data: The importance of a transdisciplinary and multidimensional approach
}

\begin{abstract}
The use of big data in higher education has evolved rapidly with a focus on the practical application of new tools and methods for supporting learning. In this paper, we depart from the core emphasis on application and delve into a mostly neglected aspect of the big data conversation in higher education. Drawing on developments in cognate disciplines, we analyse the inherent difficulties in inferring the complex phenomenon that is learning from big datasets. This analysis forms the basis for a discussion about the possibilities for systematic collaboration across different paradigms and disciplinary backgrounds in interpreting big data for enhancing learning. The aim of this paper is to provide the foundation for a research agenda, where differing conceptualisations of learning become a strength in interpreting patterns in big datasets, rather than a point of contention.
\end{abstract}

Keywords: Learning analytics, inference, predictive modelling, transdisciplinary

\section{Introduction}

While the use of big data in higher education is undoubtedly maturing, there is still some way to go before learning analytics, as an emerging discipline, reaches its potential as a collaborative endeavour. Siemens (2013, pg. 1382) described the definition of learning analytics as per the first conference on Learning Analytics and Knowledge (LAK) in 2011 thus:

Learning analytics is the measurement, collection, analysis, and reporting of data about learners and their contexts, for the purposes of understanding and optimizing learning and the environments in which it occurs.

From this definition, one of the defining characteristics of learning analytics, particularly in comparison to educational data mining, is that it is not focussed 
solely on analytical techniques (Siemens \& Baker, 2012) but on 'understanding and optimising learning'. There is, therefore, need to consider what exactly learning is and how best to infer it from large datasets in order to attempt to optimise it. This has led some in the community to emphasise that learning analytics should be about learning primarily over the analytical techniques used to monitor and intervene (Authors, 2012; Rogers, 2015). Focussing on learning as the central emphasis of learning analytics is a crucial turn for the field (Authors, 2015). Difficulties that have arisen in similar multidisciplinary fields dealing with big and complex datasets such as educational neuroscience (Authors, 2017) highlight the critical necessity of monitoring and updating assumptions and conceptualisations that underpin the analysis and interpretation of big data. Although there has been some discussion of these issues in the learning analytics literature, this paper aims to extend on these debates by providing the basis for a transdisciplinary research agenda.

We define transdisciplinarity, as per Nicolescu (2002), as a strategy for recognising the importance of what exists between and beyond disciplinary boundaries and for embracing the multiple levels of reality for a more holistic approach to understanding learning and teaching. This is important given the complexity inherent in learning theory (e.g. Jonassen \& Land, 2012) and the difficulty in defining and inferring learning from big data. We will unpack some of this complexity to suggest ways that this holistic approach might be achieved. Diverse conceptions of complex phenomena like learning \& diverse conceptions of teaching, have led to fundamental issues in other multidisciplinary activities. Recognising and developing an appreciation for these differences is important for fields like learning analytics comprising an inherently collaborative effort.

\section{Inferring learning from big data}

We begin our analysis of how learning has been inferred from data across disciplinary ontologies by first examining other areas where big data has been drawn on in an attempt to understand learning. While the availability of big data sets have created some opportunities for researchers and instructors that were not previously viable, that is not to say that there has not already been a concerted 
effort to use, particularly, behavioural, demographic and other data to understand the learning process. Psychological science, for example, has been undertaking the same task for over a century, with an emphasis on using behavioural data to infer cognitive and affective processes, making it a useful analogy for informing learning analytics. To date, there has been much focus on educational assessment (e.g. Knight, Buckingham Shum \& Littleton, 2014), measurement theory (e.g. Milligan, 2015) and psychometrics (e.g. Pardos, Baker, Gowda \& Heffernan, 2011) in relation to big data in higher education. There has been less emphasis on the parallels with the trajectory other cognate fields have taken in the learning analytics literature (Authors, 2012). There is much to be learned from the course that these disciplines have taken over the last century. There is also value in attempting to understand what can be gleaned from these disciplines to inform and leverage transdisciplinary collaborations.

One area that is of potential import is the ways in which cognate disciplines have dealt with the kinds of inferences that can be made about learning through varied methodological approaches. What is perhaps most notable for the process of inferring learning from big data is the so-called 'replication crisis' in psychological science (Maxwell, Lau \& Howard, 2015). The inability of researchers to replicate widely known and ostensibly robust observations highlights issues of statistical significance and inference. Even though experimental psychology is well-established as a sub-discipline with rigorous and reputable methodologies, there are still fundamental problems with the kinds of inferences that can be made based on the data collected. The aim of psychological experiments is to determine cause and effect relationships. In an educational context, this aligns with the goal of determining whether educational aims, interventions, designs or learning activities lead to changes in behaviour, memory or thinking. In other words, the aim is also to 'understand and optimise learning'. The central issue here is the impact of methodological and contextual issues on inference, an issue shared across analytic approaches to learning. Concerns related to sampling, generalisability, measurement error and power all speak to the underlying methodological and contextual factors that impact on learning and other psychological phenomena. More is not necessarily better; not more people, nor more variables. This has implications when analysing big data sets. More 
people can inflate power and make it appear that there is a meaningful (and statistically significant) effect being observed, but there may be no corresponding influence on the size of the effect (Wise \& Shaffer, 2015). Similarly, adding more variables increases the likelihood of covariance between the factors of interest and raises the possibility that constructs are not being adequately assessed. Operationalisation of variables in this context becomes increasingly difficult, particularly when seen through the lens of a specific discipline. On the one hand, highly specific studies examining individual variables in subgroups have limited generalisability that can be artificially inflated due to increases in power without associated increases in effect size. On the other hand, increasing the number of variables introduces substantial noise and covariance. This tension between rigour and relevance is common across research on educational technologies (e.g. Reeves, 2006).

The dilemma that psychological science currently finds itself in has implications for the interpretation of big data in higher education. Learning analytics offer the potential for large-scale analysis, particularly in MOOCs (Milligan, 2015). Large numbers allow for multilevel analysis across many variables and temporal, situational and event-related contexts. The power inherent in these enormous sample sizes and potentially substantial numbers of variables across time, location and settings carry an additional conceptual problem that cannot only be solved by statistical methods alone. As such, we cannot appropriately justify data-driven inference. How does one ensure meaningful inferences are made from data that are more likely to be significant based on the power within the statistical model? Power is often considered a source of measure of acceptance or establishing of the generalisability of the findings of research. This statistical argument is insufficient in regular research, as other pertinent issues also compound the criteria for generalisability, for example, sampling bias. The limitations of statistical models for determining what is occurring in the mind are therefore becoming apparent and do not lend themselves to being easily resolved by increasing sample sizes or the number of variables being examined. Data-driven inference, therefore, does not appear to be sufficient to provide a clear picture of psychological \& social phenomena such as learning. This complication has implications when considering the use of big data for enhancing learning in higher education, 
particularly given that researchers with different disciplinary backgrounds will interpret the same data in different ways.

\section{Learning: process or outcome, or both?}

The issues evident in psychological science provide the basis to delve deeper into the problems around inferring learning from big data. Even if we are to accept the assumption that learning occurs in the mind (which is controversial enough), the fundamental construct is multi-dimensional, content specific and contextdependent. This includes both the context in which the data is collected and the epistemic context through which a researcher attempts to make sense of the data. De Houwer, Barnes-Holmes and Moors (2013) are critical of research examining learning, making the argument that it is often not even clear in research whether learning is being treated as a process or outcome. Following this, Sodestrom and Bjork (2015) contend that what is often conceptualised as learning in research and practice is, in reality, performance. While educationally, learning has been traditionally seen as a developmental process, in recent times, this idea has also been conflated with performance. The increased emphasis on graduate attributes and work-ready skills suggests a shift towards performance in higher education, such an interpretation of learning is some way from how a psychological or learning scientist would view learning. University graduates are now being assessed on what they can do (in real or simulated settings), rather than who they are or how they have and will continue to develop as emerging professionals, scientists or scholars. The implication of these trends is that there is an emphasis on a snapshot of performance as a means of determining student learning rather than seeing learning for what it is, a developmental process leading to an (ongoing) outcome. The confusion in even this most foundational definition of learning highlights the potential mismatch in the kinds of interpretations different researchers with different disciplinary backgrounds will make on the basis of the same data.

This misguided focus on performance over learning therefore masks a deeper issue about what learning is. Jacobson, Kapur and Reimann (2016) discuss a shift in the focus of educational research on learning towards more complex, systems- 
based conceptualisations. This change is an attempt to move the debate forward from the clear divide between cognitive and social/situated conceptions of learning. Their premise is that complex, systems-based models can incorporate both. Nathan and Alibali (2010) further argue that the learning sciences should be engaged in complementary activities featuring both elemental (foundational cognitive psychology-type work) and systematic work that addresses more complex, situated issues. Their reasoning for making this argument is that learning occurs across multiple spatial and temporal dimensions. In both articles, the authors highlight the difficulty in conceptualising and attempting to enhance learning. These are fundamentally complex tasks, particularly due to the difficulty in translating basic learning processes up to authentic and socially complex learning environments, as encountered in real-world and immersive contexts.

The complications inherent in trying to infer learning from data are due to both the distal nature of the phenomenon in question from the instruments attempting to measure it and the large variability in processes and outcomes. There is, therefore, great difficulty in using analytic approaches that have proven useful for making meaning from big data in other industries. For example, both marketing models (in Business fields) and epidemiological models (arising from Medicine) have advantages in that they can rely on the use of natural experiments (see Dunning, 2008) to infer causal, moderating and mediating factors underpinning economic or health phenomena. However, while those contexts have been drawn on to think about education settings, both provide poor analogies for interpreting big data in higher education. Marketing as a model for learning analytics fails because of the dualist nature of the outcome (buy/do not buy - click/do not click etc.). Similarly, epidemiological analogies do not work because the disease model tends to shift the outcome focus in two dimensions; morbidity and mortality. Again, the results on both dimensions tend to be binary; people are ill or not ill, dead or not dead. In higher education contexts, we mitigate against binaries that may cause to polarise, segregate and stereotype learners and their learning.

Attempting to model and make decisions based on binary outcome data like this has been most famously criticised as an example of the 'McNamara fallacy' (Basler, 2009). This fallacy is named after a Vietnam War era US Secretary of 
Defence who over-relied on crude quantifications such as total numbers of casualties to determine progress in the war. These crude measures did not adequately capture the complexity of the war, and ineffective strategies were employed thus. Similarly, learning is multidimensional, complex and requires the preparation of graduates for an unknowable future (Barnett, 2004). This complexity is apparent in ongoing work in assessment design and psychometrics. Both areas have an extensive body of literature aimed at determining how best to tell whether students have learned and whether they are developing the knowledge, skills and abilities for their future needs. Furthermore, there is heated debate about whether the sorts of outcomes being achieved by college students are adequate for the future workforce (Arum \& Roksa, 2011), and whether indeed, such outcomes should be the purpose of higher education at all (Authors, 2016). If learning outcomes were easy to operationalise and measure, there would be less need for such work or critique.

While there is a danger inherent in attempting to infer a phenomenon as complex as learning from simple data in big datasets, there is a second aspect of these inferences that warrants mention here. Simplifying complex outcomes into relatively straightforward measures also risks invoking Campbell's law (Nichols $\&$ Berliner, 2007). This law is captured most concisely in the saying 'teaching to the test' that was applied to standardised national testing in primary and secondary education. In other words, simplified output measures become the goal of education rather than the earlier focus on teaching for quality learning. This perversion of the purpose of learning creeping into university settings is particularly problematic as it perpetuates an industrialised version of education. Students as graduates risk being seen as products for industry, the measures used to monitor their performance give a sense of how well they are prepared to work, rather than how they have developed and can contribute a critical disposition as educated citizens. As Rowntree (1987) argues, inherent in both the McNamara fallacy and Campbell's law are bias towards quantification that implies that anything that cannot be measured is not worthwhile. The measures we currently have do not capture the complexities and multidimensional nature of learning, despite the nascent potential of large datasets. The issues humanity faces in the $21^{\text {st }}$ Century, therefore, require more sophisticated approaches to learning as 
ongoing development, rather than what can most easily be quantified to the satisfaction of economic rationalism and other interests.

It is perhaps a truism to argue that learning, as a construct, ranges from repetition to higher order, complex phenomena, and must always be considered within the interplays of all historical, cultural, political, economic and social contexts that it occurs in. Beyond this however, that there is seemingly no consensus whether learning should be considered as a process or outcome, or both, suggests that there are many (conflicting) assumptions teachers, researchers and practitioners bring to learning analytics as they do to cognate endeavours attempting to understand and enhance learning. These assumptions are untapped, currently not routinely explored, which may create a barrier for genuine transdisciplinary thinking and actions within learning analytics research.

\section{Complex phenomenon - complex model?}

One possible account for the complex mix of conceptualisations of learning is to consider using much more sophisticated models that attempt to capture better the complexity of learning from large datasets. Jacobson et al. (2016) argue that to continue to make progress in understanding and enhancing learning, it is indeed in complexity science that answers are to be found. A complex phenomenon requires a paradigm that has complexity at its core. Intelligent tutoring systems (ITS) provide a good example demonstrating how difficult and complex it is to create efficient statistical models of student learning (Mislevy \& Gitomer, 1996). Intelligent tutoring systems have been in development for decades since the pioneering work of Newell (1990). The design of these systems, while impressive, is still some way off providing truly intelligent and adaptive tutoring. Holland, Holyoak, Nisbett, and Thagard (1986) made the argument that it is challenging to codify the inferential processes that humans are capable of into a machine. More recently, Baker (2016) pointed out that ITS remain 'stupid' in comparison to the people they are designed to assist. Instead, Baker argues that systems should be built to augment, rather than supplant human capabilities. 
Although modelling approaches used to infer learning from big datasets use a combination of inductive and deductive logic, there is always, intrinsically, an inferential gap between human and machine. Statistical prediction cannot, no matter how large or complex the dataset, remove the need for inference and interpretation (see also Holland et al., 1986). This requirement means that there are limited options concerning how to progress with these predictions. One is to continue to tweak the model through iterations. This process occurs already measurement models, predictive analytics and so on. Iterative cycling through model updating (or learning) is foundational for neural network models (particularly Hebbian-based models) and machine learning (Strickland, 2014). There are undoubtedly significant advances being made in increasingly complex and adaptive models and algorithms, that is not in dispute. Whether these sophisticated modelling approaches can seamlessly bridge the gap between datadriven inference and complex, messy reality is unclear. So long as there remains a gap, there will also be alternative interpretations about how best to infer across the gap. The assumptions being made about this meaning making, inferential process therefore remain problematic.

Alternative methods for predictive modelling try to understand better the assumptions being made by referring to theory (Authors 2012a). Conversations and ongoing collaboration between the technical and theoretical communities will help to bridge the research and practice gap and lead to better inferences. Ideally, though, both the iterative improvement of models and the benchmarking of the models against complex reality are used in unison. Whether that has been true of the field of learning analytics is up for debate. To date, there has been more focus on educational practice, intervention and the interface between big data, analytics and design (ethical issues aside). It is fair to say that there has been less focus on determining what it is that is the actual phenomenon of analysis - learning (Liu, Rogers \& Pardo, 2015; Authors, 2012) and what kinds of assumptions about how best to infer learning research from different disciplines and traditions bring to that analysis. 


\section{Inference and translation of big data in practice}

Learning analytics as a field can learn much from the well-worn path that psychological science, educational neuroscience and to an extent, educational technology (see Jones \& Kennedy, 2011) have taken when attempting to use data to inform practice. While there may be no replication crisis of the sort psychological science is experiencing in the use of big data in higher education, it can reasonably be expected that there will be due challenges and upheavals ahead. Like the replication crisis, these will inevitably link to fundamental issues of rigour and validity due to the diversity of approaches different researchers bring to the field based on their academic and contextual backgrounds. Learning analytics, as a developing field, may not be well prepared to address these changes due to the emphasis to date on the practical aspects of learning analytics implementation, as has often been the case in similar interdisciplinary projects.

Since its inception, learning analytics as an educational science field has been inextricably linked to educational practice. Learning analytics is a transdisciplinary area of research by default, as it is grounded in transdisciplinary educational practices. With growing availability and access to digital big data sources, universities and educators increasingly see the lure of its affordance in enhancing student retention, learning experiences, and instructional practices. This attraction has resulted in the increasingly emerging view that the integration of learning analytics in industry-wide educational practice (from classroom levels to institutional, to national levels) as impending or inevitable (Authors, 2014). This perception seems to have occurred despite there being no established path forward for translation of basic research into practice and vice-versa. Whilst there are and may be many paths, a research-informed approach necessitates the breaking down of barriers between levels of analysis, to allow translation to flourish .

Challenges to institutional adoption aside, herein lay the issue: the growth in use of big data in practice parallels that of the growth in big data research - a doubleedged sword in evidence-based educational practice. While practitioners are eager to adopt big data to enhance student learning and teaching practices, the codevelopment of an emerging evidence base to inform practice effectively means 
that there are immediate challenges to translation for practice. It is clear that analytics can, has, and will open doors for process level analyses in digital learning and education. Like other learning science methods, and theories of practice, learning analytics is not impervious to considerations of design rigour and relevance to facilitate causal inferences. While the ease of access to the learning analytics (raw source log data, transformed, aggregated, or algorithmic) has relatively increased, it simply is not a substitute for deep thinking across different perspectives. Rather, critical reflection in using big data in higher education practice contexts becomes even more necessary.

With the affordances that the analysis and interpretation of big data bring therefore come significant translational, inferential and implementation challenges. The impact of inferential implications is more pronounced given the temporal, conceptual, and logistical proximity to practice in higher education. There are a few specific issues of concern relating to the challenges with inferences about behaviours from learning analytics that directly impact educational practice. Learning analytics is conceptualised as a sense-making and actionable science in practice (Authors, 2015; Siemens, 2013). That is, once you have access to data, whether visualised or analysed, this should lead to better understanding of the learning processes in question and subsequently, design of actions to improve student learning or instructional practice in that domain. The issues of inference, therefore, become fundamental to the transdisciplinary, translational processes in sense-making. While critical discussions of translational issues in inferences made from big data have emerged (Reimann, 2016; Wise \& Shaffer, 2015), learning analytics as a practice-focussed field could further benefit from the lessons learnt in the health and clinical sciences, which have been able to achieve a significant level of transdisciplinarity. Considerations are evident for valid inferences, translation, and implementation in practice to facilitate our progress in bridging research and practice. Green and Glasgow (2006) suggested that over-reliance on decontextualised implementation of clinical research have limited the rate of integration of evidence into health practice. Consequently, they have recommended evaluation criteria for researchers and practitioners in a bid to facilitate the translation of research to practice (i.e., RE-AIM, Reach, Effectiveness, Adoption, Implementation, Maintenance). The framework has also 
been demonstrated as a useful means to conduct systematic reviews and metaanalyses across complex and broad health science research (e.g., Blackman et al., 2013; Laranjo et al., 2014). Equally important is the assessment of evidence-based practice. For example, while an evaluation framework has been developed, used widely, and demonstrated to be useful in facilitating successful implementation of evidence-based practice (Kitson, Harvey \& McCormack, 1998), the evaluation of the efficacy of such frameworks, nor the capacity to refine the frameworks have yet been realised (Kitson et al., 2008). As a genuinely transdisciplinary research and practice field such as learning analytics, two-way communication between researchers and practitioners is crucial to advance empirical and theoretical development in complex, collaborative research and practice contexts.

Another implication of inferential challenges for the use of big data in higher education is towards actionable science. Inherent in linking learning analytics to action in practice is the impact of learning analytics practice on students; for their experiences, learning, motivation, engagement and their lives in general. These complex considerations that need to be factored in when making inferences lie in addition to issues identified in a related field, data-driven decision making in schools (Datnow \& Hubbard, 2016; Farrell \& Marsh, 2016). That is, in driving action, data is sometimes viewed as evidence in and of itself and thus may be perceived as sufficient in justifying directions for actions. This illusion of objectivity of data or analytics is a cognitive fallacy (Burger \& Berry, 1988), and can result in a false sense of rigour (Dover \& Schultz, 2016) and potential misapplication of sense-making and actions (Cohen, 1994). This perception of data as unquestionably objective has been shown to be problematic in other domains. For example, the claimed objectivity of big data mining in drug safety has been demonstrated to be a barrier to effective pharmacological clinical judgement. Outcomes from different software systems and vendor tools were deemed to be discrepant simply through differential data transformation and computation methods (Hauben, Reich, Gerrits \& Younus, 2007). This result, therefore, prompted the critical role of collaborative communication between the technical and functional subdomains, facilitating more realistic perceptions of the data. This approach was beneficial for informing clinical analysis and decision making to realise the advantageous utility of the algorithmic support system. 
Acknowledging the role of relative subjectivity and complexity in making inferences could not only open the way for more accurate and appropriate judgement and decision making but also recognises the realities of the complexities of measurement and meaning making. Thus, understandably, the focus on issues about ethics in using learning analytics is prevalent in affecting educational practice (e.g., Ferguson, Hoel, Scheffel \& Drachsler, 2016; Prinsloo $\&$ Slade, 2016). How do issues in making inferences from big datasets impact on ethics? We propose that understanding these inferential issues in complexity and being more explicit about the assumptions and potential biases beneath the inferences is fundamental to ethical learning analytics practice and application. Increased attention to the assumptions beneath the complexity necessitates more rigorous judgements of the defensibility of the inferences and proposed or designed actions. Buckingham Shum and Crick (2016) suggest that engaging with this complexity is inevitable for the sector to achieve the unique challenges learning analytics as a field is working towards overcoming.

Practitioners have the added responsibility of critically considering the consequences of their actions beyond intended improvements in learning, teaching, or retention, to that of impact on individuals and groups, as well as the emergent ecological consequences. While data-driven action is often wellintentioned, fundamental attribution or inferential errors made when analysing big data in practice can impede positive transformational change in application. That is, interventions or actions designed based on incorrect inference arguably would result in lower likelihoods of implementation success as the assumptions underlying the model are flawed, thereby limiting the potential benefit of learning analytics in enhancing educational practice. By the same token, when care is taken with the inferences made, the potential of learning analytics in improving practice becomes tangible. Recent evidence suggests that the power of evidence-based, well-informed actions and interventions does not simply end with individuals; people who have benefited can improve their social environments thereby helping others indirectly (Powers et al., 2016). Thus, prioritising considerations for shared transdisciplinary approaches and deliberate inferential processes over the impetus for obligation to act, would facilitate the successful implementation of welldesigned interventions. 


\section{A transdisciplinary approach to interpreting big data}

So far in this paper, we have attempted to delve into some of the fundamental issues about how learning is conceptualised and inferred broadly about big data and what this means in practice. We have also highlighted some of the unresolved issues related to inference that could be considered more deeply by the community. We now turn our attention forward. Our aim in doing so is to attempt to move beyond the theoretical towards practical means of enhancing communication and cooperation between the different ways of being brought to the community by various disciplinary groups. Careful consideration of disciplinary differences in ontology and epistemology has also been problematic in other cognate fields such as educational neuroscience. However, a concerted effort to bridge the laboratory and the classroom (see Authors, 2017a) is beginning to move educational neuroscience towards a transdisciplinary research agenda. Previous attempts at constructive collaboration between computer science, psychology and philosophy such as the pioneering work of Holland and colleagues (1986) have been highlighted as indicative of the substantial benefits on offer when diverse disciplines work in harmony rather than in opposition (Smoliar, 1987). We hope to provide a stimulus here towards the same goal in the interpretation of big data for enhancing learning in higher education.

One difficulty in working across disciplines that is evident for example in educational neuroscience is that there are multiple layers of interpretation and analysis of learning from small parts of the brain over minute timeframes to the whole student over a lifetime (see Authors, 2017). By framing levels of analysis and action into abridged conceptual models, a possible way forward towards bridging these layers can be proposed. The primary means of allowing for meaningful translation and transdisciplinary collaboration is to make explicit the assumptions underpinning the inferences being made about learning on the basis of the data. The remainder of this article reflects models for representing ways of approaching sense-making and how these impact on meaningful translation, inference and thus, transdisciplinarity across levels of analysis. Borrowing from the progress that has been made in educational neuroscience, there appear to be 
two main options for explicating these assumptions and inferences across different levels of analysis. The first is to continue to assume a linear, hierarchical structure and cut down level of inference by conducting research and carefully interpreting the results incrementally between layers or levels of interpretation. This approach is similar in many regards to that represented in Bloom's revised taxonomy (Krathwohl, 2002). Using Ackoff's (1989) representation of the path from data to wisdom, what this means is that each stage of the process involves a deliberate process of inference, translation and meaning-making (see figure 1).

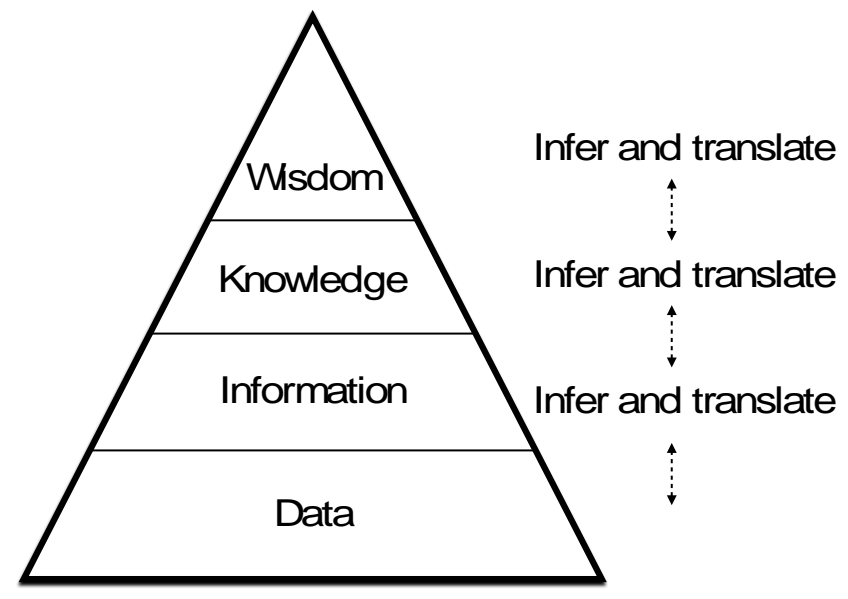

Figure 1. Representation of the Data, Information, Knowledge, Wisdom framework (adapted from Ackoff, 1989)

When compared with the research and translation process that has been suggested for educational neuroscience, it is then possible to begin to see what a linear translation process might mean for interpreting big data in higher education. The process for educational neuroscience has been adapted from Horvath and Donoghue (2016). Like that of Ackoff (1989) the aim here is to make explicit the assumptions leading to the level of inferences being made, then break down the inferential distance into closer steps (see figure 2). So, a finding from a neuroimaging study can have impact on teaching practice in the classroom if it is adequately interpreted at the level of the brain and mind, through the behavioural level and again reinterpreted for teaching practice. Such a research agenda involves neuroscientists, cognitive psychologists, educational psychologists and teachers all working together across levels of data collection and interpretation. 


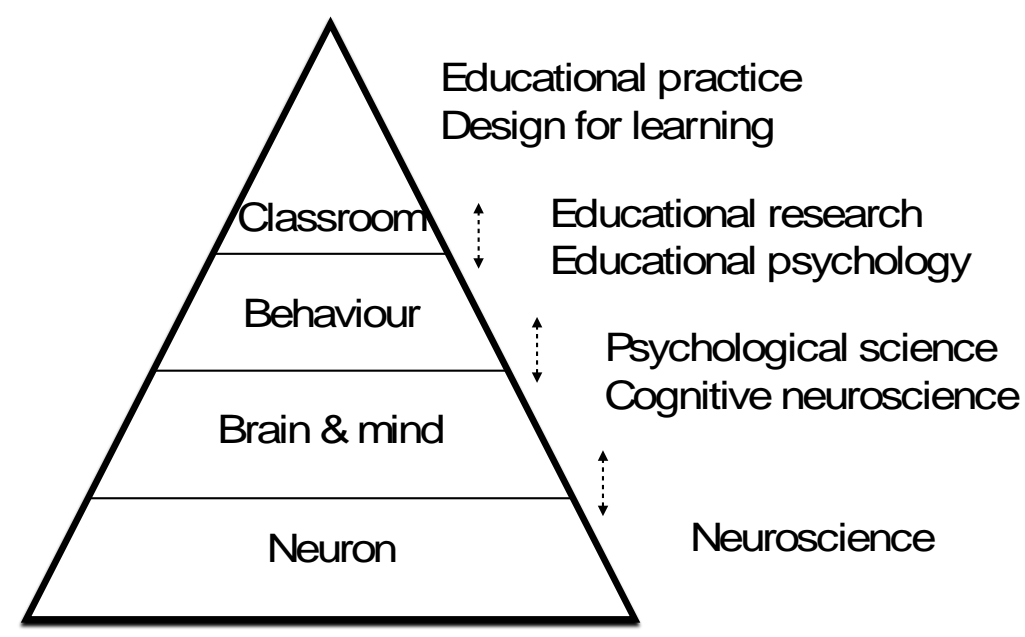

Figure 2. Representation of linear translation process in educational neuroscience (adapted from Horvath \& Donoghue, 2016)

Building on an earlier version of a similar translational hierarchy (Authors, 2012), it is possible to extend on these previous models and come up with a version for situating big data analysis in the context of higher education research. Such a hierarchy is presented in figure 3. Again, this depiction highlights the need to make meaning of data collected through research conducted at each level for the other levels. The aim is presented here as having an impact on students as individuals as they exist in the world.

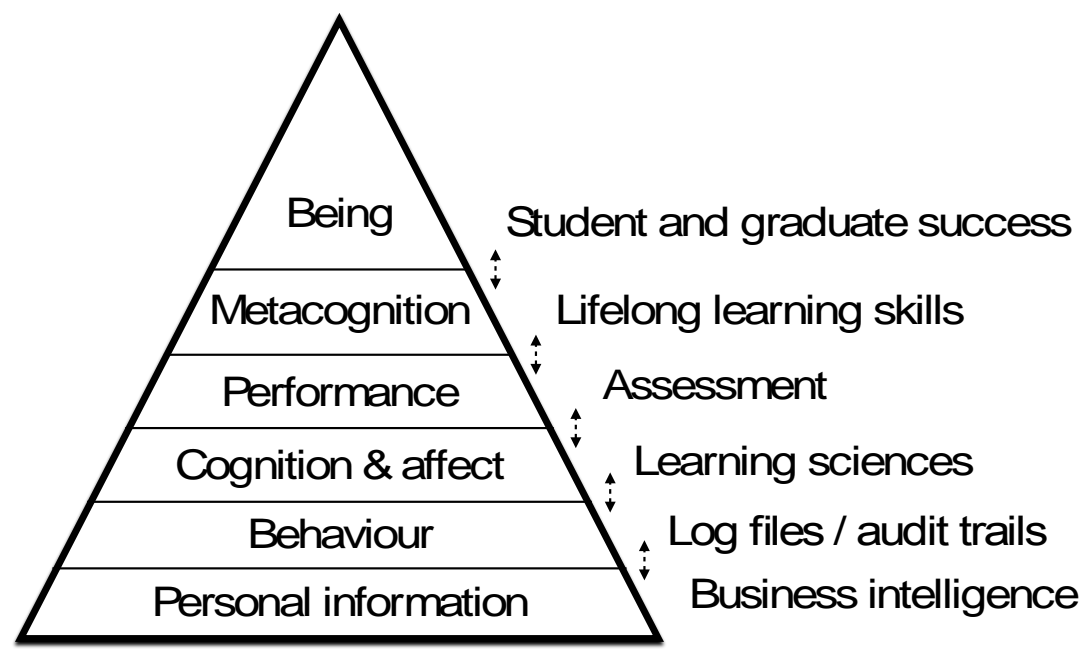

Figure 3. Representation of a linear translation process for big data interpretation (adapted from Authors, 2012)

We will not dwell on the ontological nature of the hierarchy as we have constructed it, a fuller discussion of the reasons why it is important to impact on both higher cognitive skills, like metacognition and on student being in the world 
can be found in Authors (2012). The aim here is simply to suggest a possible linear translation and analysis approach for research on learning and how it can help interpretation of big datasets. To assist with that aim, we have also added to this figure an indication of where the information at each level is to be found. This model, therefore, underpins a possible agenda for research into aspects of learning in higher education where a particular aspect of learning. For example, student misconceptions, can be examined in small-scale, controlled environments, and then extended to more realistic, complex learning environments. The small-scale studies, in this case, would generate hypotheses about what kinds of behaviours are most likely to indicate that a student has a misconception. Translation of this sort means that the inferences being made about the patterns evident in large reallife datasets are grounded in empirical evidence from laboratory studies. Therefore, any inferential gaps emerging from the interpretation of big data alone can be explicitly tested under controlled conditions.

The linear translation approach is one way of systematically creating a framework for collaboration across levels of inference in learning analytics. A second option, however, is to implement something akin to an ecosystem model. A representation of this notion is presented in figure 4 .

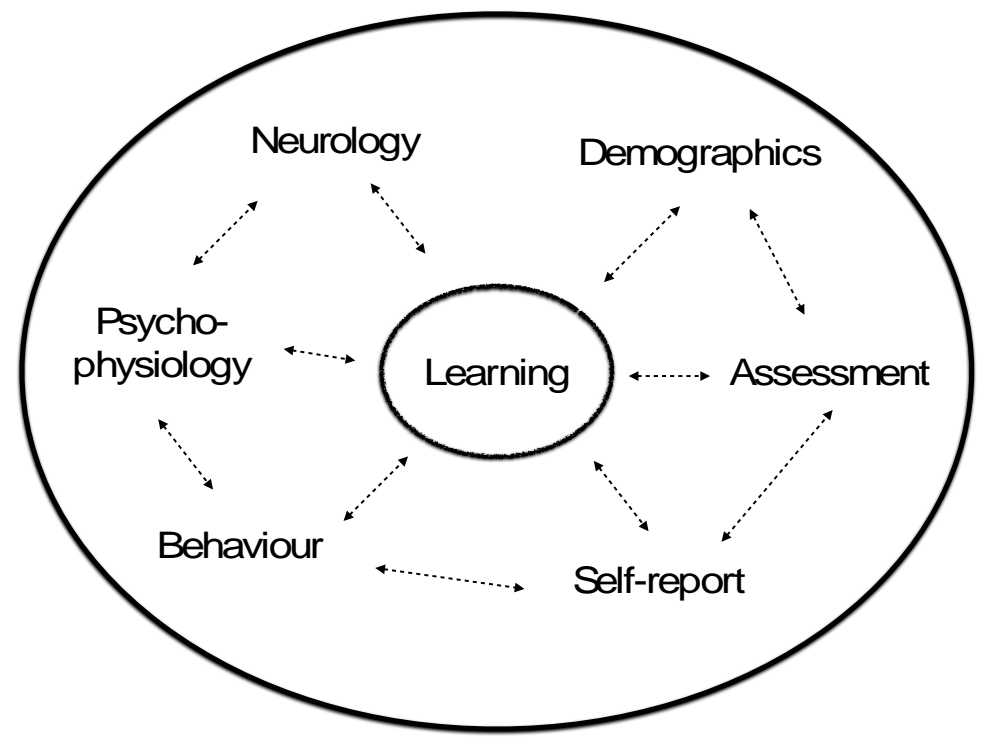

Figure 4. Representation of an ecosystem model of evidence for big data interpretation

This approach would involve a meta-model of learning where many inferences are made and triangulated. This model has been advocated in the emerging field of 
psychoinformatics (Yarkoni, 2012). A feature of this approach is that, rather than treating different forms of information as hierarchical in time and space, all indicators and conceptualisations are treated as broadly equal and indicative of the central and holistic meta-construct. While this ecological approach may suggest some issues about conceptual confusion, many of these matters are dealt with using measurement models and sophisticated statistical inference. Bayesian modelling and machine learning are already making inroads in this regard. Diverse forms of evidence can be built into these models, and they can be refined over time as more information is passed through the system. Weightings can be manipulated based on what seems to be impacting on the outcomes the most. From this, a more sophisticated model of learning can be developed that provides improved fit to the data.

In practice, this model could inform the application of learning analytics across levels of analysis and a diverse set of researchers with different methods for interpreting data. To add to the complexity, these levels of analysis and interpretation is situated in dynamic complexity within the higher education system. While the larger system complexity is out of scope of this paper, the explication of approaches to conceptions of learning at the outset may at the very least more firmly contextualise directions in transdisciplinary collaborations. As we have alluded to in thus far, the key here is that all members of a transdisciplinary project discuss and make explicit their conceptual resources: that is, their definition of learning, where they see learning occurring and the methods by which they infer learning from the outset. A hierarchical model belies an assumption that some forms of evidence are more rigorous or relevant than others. While this may be true to an extent, it does not help resolve fundamental differences in the approach that researchers and practitioners from different disciplines to make sense of data. Our ecological model is not designed to be an exact representation of reality, however, it does provide a way of thinking about evidence that moves beyond a notion that some forms of evidence are superior to others.

While there is immense promise in discussions that allow for all forms of evidence to be considered, they have not necessarily helped to resolve problems in 
neuroscience, in behavioural economics or medicine. When all data are treated as qualitatively equal and allowed to drive the process, there is less space left for the data to have meaning to teachers or students. When implemented back into the complex social milieu the process ceases to be effective, however, some progress can at least be made towards breaking down disciplinary boundaries.

There are challenges with implementing either a linear or ecosystem-like approach to systematic, collaborative research outlined here. These problems lend credence to the argument made by Jacobson et al. (2016) that systems-based approaches may be a better option. The difficulty in adopting a systems-based approach is that it requires a level of expertise in systems thinking. Validity will continue to be an issue in interpreting big data in higher education no matter how collaboration occurs, whether that be within a linear, ecosystem or system-based framework. There is also some uncertainty as to whether different conceptualisations of learning create an insurmountable hurdle to systematic collaboration. For example, situated views of cognition and learning look to the complex physical and social context in which learning occurs to determine how it happens (Anderson, Reder \& Simon, 1996). Cognitivist views focus on what takes place in the mind of the individual. Equating these two different notions about the location of learning make working across these differing interpretations difficult despite the arguments made by Jacobson et al. (2016) and Nathan and Alibali (2010). Both paradigms look for evidence of learning in very different places and consequently offer unique insights/ perspectives on learning. We have attempted here to provide some guidance as to how these sites may relate to each other.

There are clear benefits of a research agenda that involves the explicit discussion about the assumptions that researchers make about learning when interpreting learning analytics data. A more systematic means of translation and implementation from each of the various views on the location and mechanisms of learning enables multiple affordances within a complex system (as per Holland et al., 1986). In educational neuroscience, this is beginning to allow for the inferences being made to have an impact from the laboratory to the classroom and online learning environment and back again (Authors, 2017). To date, learning analytics has predominantly focussed on click streams and log data that are 
relatively easy to collect but have limited utility in understanding learning deeply (Liu et al., 2015). Having a systematic way of fostering these conversations both builds on and generates the affordances of multiple pathways for understanding learning. We are therefore in agreement with Nathan and Alibali (2010) that any endeavour broadly characterised as the learning sciences should involve complementary systematic and elemental aspects. In other words, translation goes both ways; research systematically informs practice and practice routinely informs research. Referring to our earlier example, misconceptions observed 'in the wild' through big data in MOOCs or learning management systems can help to inform the kinds of studies carried out on misconceptions in the laboratory. These studies, in turn, provide a sharper focus for the exploration of patterns evident in big datasets. This translational approach may not resolve any issues surrounding the conceptual complexity of the phenomenon. However, it may at least give everyone attempting to infer learning from big data, no matter their discipline or role, a means to converse about the kinds of inferences we can make about data and what they tell us about student learning.

\section{CONCLUSION}

The issues we have discussed in this article are not unique to learning analytics. In the learning sciences, there is continuing debate about what learning is and how best to infer it. Theoretical and methodological isolation of work does not productively engage researchers in much-needed transdisciplinary approaches. In an attempt to resolve these debates, researchers and practitioners cannot operate solely in their disciplinary silos. To move the learning analytics field forward, a transdisciplinary approach needs to be crafted, exploited and adopted where differences in ways of thinking, being, doing and valuing become points of strength, rather than points of contention. Critical to this will be an ongoing testing of the assumptions that are made about the validity of the data collected and what it is each individual and sub-discipline are actually referring to when speaking of learning and their conceptions of teaching. The lessons of psychological science and educational neuroscience suggest that privileging particular forms of knowing, or specific professions over others in this endeavour risk overextending the inferences made based on the data at hand. 
While it might be ideal for each person engaged in researching or implementing learning analytics to have expertise across all the relevant areas, this is not realistic at this point. Although aspirational, it may also be difficult for systemsbased models of learning to underpin the ongoing collaboration between stakeholders attempting to make sense of big datasets in the higher education context. The complexity of our task means that more efficient ways of coming together and facilitation that moves the conversation forward are needed. This initiative includes, and indeed is premised on, being mindful of the epistemological and ontological realities of collaborators, researchers and practitioners in the field. While each may not all see learning in the same way, and try to infer learning using different methodologies, we have attempted in this paper to provide necessary ground to move the transdisciplinary conversation forward and provide a basis for a multidimensional research agenda including deliberate sense-making and translation across levels of inference.

\section{REFERENCES}

Ackoff, R. L. (1989). From data to wisdom, Journal of Applied Systems Analysis 16, 3-9.

Anderson, J. R., Reder, L. M., \& Simon, H. A. (1996). Situated learning and education. Educational Researcher, 25, 5-11.

Arum, R., \& Roksa, J. (2011). Academically adrift: Limited learning on college campuses. Chicago, IL: University of Chicago Press.

Authors (2012).

Authors (2012a).

Authors (2014).

Authors (2015).

Authors (2016).

Authors (2017).

Authors (2017a).

Authors (under review).

Baker, R. S. (2016). Stupid tutoring systems, intelligent humans. International Journal of Artificial Intelligence in Education, 26(2):600-614. 
Barnett, R. (2004). Learning for an unknown future. Higher Education Research \& Development, 23(3), 247-260. doi: 10.1080/0729436042000235382

Basler, M. H. (2009). Utility of the McNamara fallacy. BMJ. 339: b3141. doi: 10.1136/bmj.b3141

Berger, J. O., \& Berry, D. A. (1988). Statistical analysis and the illusion objectivity. American Scientist, 76, 159-165.

Blackman, K. C. A., Zoellner, J., Berrey, L. M., Alexander, R., Faning, J., Hill, J. L., \& Estabrooks, P. A. (2013). Assessing the internal and external validity of mobile health physical activity promotion interventions: A systematic literature review using the RE_AIM framework. Journal of Medical Internet Research, 15(10), doi: 10.2196/jmir.2745.

Buckingham Shum, S. \& Crick, R. D. (2016). Learning analytics for 21st century competencies. Journal of Learning Analytics, 3(2), 6-21

Cohen, J. (1994). The earth is round (p <.05). American Psychologist, 49 (12), 997-1003.

Datnow, A. \& Hubbard, L. (2016). Teacher capacity for and beliefs about datadriven decision making: A literature review of international research. Journal of Educational Change, 17 (1), 7-28.

De Houwer, J., Barnes-Holmes, D., \& Moors, A. (2013). What is learning? On the nature and merits of a functional definition of learning. Psychonomic Bulletin \& Review, 20(4), 631-642. doi: 10.3758/s13423-013-0386-3

Dover, A. G. \& Schultz, B. D. (2016). Troubling the edTPA; Illusions of objectivity and rigor. The Educational Forum, 80 (1), 95-106.

Dunning, T. (2008). Improving causal inference: strengths and limitations of natural experiments. Political Research Quarterly 61, 282-93.

Farrell, C. C. \& Marsh, J. A. (2016). Contributing conditions: A qualitative comparative analysis of teachers' instructional responses to data. Teaching and Teacher Education, 60, 398-412.

Ferguson, R., Hoel, T., Scheffel, M., \& Drachsler, H. (2016). Guest editorial: Ethics and privacy in learning analytics. Journal of Learning Analytics, $3(1), 5-15$.

Green, L. W. \& Glasgow, R. E. (2006). Evaluating the relevance, generalization, and applicability of research: Issues in external validation and translation methodology. Evaluation \& The Health Professionals, 29(1), 126-153. 
Hauben, M., Reich, L., Gerrits, C. M., \& Younus, M. (2007). Illusion of objectivity and a recommendation for reporting data mining results. European Journal of Clinical Pharmacology, 63, 517-521.

Holland, J., Holyoak, K., Nisbett, R., \& Thagard, P. (1986). Induction: Processes of inference, learning, and discovery. Cambridge, MA: MIT Press.

Horvath, J. C. \& Donoghue, G. M. (2016). A bridge too far-revisited: Reframing Bruer's neuroeducation argument for modern science of learning practitioners. Frontiers in Psychology 7: 377.

Jacobson, M. J., Kapur, M., \& Reimann, P. (2016). Conceptualizing debates in learning and educational research: Toward a complex systems conceptual framework of learning. Educational Psychologist, 51 (2), 210-218. doi: $10.1080 / 00461520.2016 .1166963$

Jonassen, D., \& Land, S. (2012). Theoretical foundations of learning environments. Abingdon, UK: Routledge.

Jones, C. \& Kennedy, G. (2011). Stepping beyond the paradigm wars: pluralist methods for research in learning technology. ALTC 2011 Proceedings of the 18th international conference of the Association for Learning Technology, University of Leeds, UK, 6-8 September, pp. 18-28.

Kitson, A. L., Harvey, G., \& McCormack, B. (1998). Enabling the implementation of evidence based practice: A conceptual framework. Quality in Health Care, 7, 149-159.

Kitson, A. L., Rycroft-Malone, J., Harvey, G., McCormack, B., Seers, K., \& Titchen, A. (2008). Evaluating the successful implementation of evidence into practice using the PARiHS framework: theoretical and practical challenges. Implementation Science, 3 (1), doi: 10.1186/1748-5908-3-1.

Knight, S., Buckingham Shum, S. \& Littleton, K. (2014). Epistemology, assessment, pedagogy: Where learning meets analytics in the middle space. Journal of Learning Analytics 1 (2): 23-47.

Krathwohl, D. R. (2002). A revision of Bloom's taxonomy: An overview. Theory into Practice, 41(4), 212-218. doi:10.1207/s15430421tip4104_2.

Laranjo, L., Arguel, A., Neves, A. L., Gallagher, A. M., Kaplan, R., Mortimer, N. \& Lau, A. Y. S. (2014). The influence of social networking sites on behavior change: A systematic review and meta-analysis. Journal of the American Medical Informatics Association, 0, 1-10. 
Liu, D.Y-T., Rogers, T., \& Pardo, A. (2015). Learning analytics - are we at risk of missing the point? In T. Reiners, B.R. von Konsky, D. Gibson, V. Chang, L. Irving, \& K. Clarke (Eds.), Globally connected, digitally enabled. Proceedings ascilite 2015 in Perth.

Maxwell, S. E., Lau, M. Y., \& Howard, G. S. (2015). Is psychology suffering from a replication crisis? What does "failure to replicate" really mean? American Psychologist, 70(6), 487.

Milligan, S., (2015. March). Crowd-sourced learning in MOOCs: learning analytics meets measurement theory. In Proceedings of the Fifth International Conference on Learning Analytics and Knowledge (pp. 151155). ACM.

Mislevy, R. \& Gitomer, D. (1996). The role of probability-based inference in an intelligent tutoring system. User-Mediated and User-Adapted Interaction, 5, 253-282. doi:10.1037/e652102011-001

Nathan, M. J., \& Alibali, M. W. (2010). Learning sciences. Wiley Interdisciplinary Reviews: Cognitive Science, 1(3), 329-345.

Newell, A. (1990). Unified theories of cognition. Cambridge, MA: Harvard University Press.

Nichols, S. L. \& Berliner, D. C. (2007). Collateral Damage: How High-Stakes Testing Corrupts America's Schools. Cambridge, MA: Harvard Education Press.

Nicolescu, B. (2002). Manifesto of transdisciplinarity. Albany, US: State University if New York Press.

Pardos, Z. A., Baker, R. S., Gowda, S. M., Heffernan, N. T. (2011). The sum is greater than the parts: Ensembling models of student knowledge in educational software. SIGKDD Explorations, 13 (2), 37-44.

Powers, J. T., Cook, J. E., Purdie-Vaughns, V., Garcia, J., Apfel, N., \& Cohen, G. L. (2016). Changing environments by changing individuals: The emergent effects of psychological intervention. Psychological Science, 27(2), 150160.

Prinsloo, P., \& Slade, S. (2016). Student vulnerability, agency, and learning analytics: An exploration. Journal of Learning Analytics, 3(1), 159-182. 
Reeves, T. (2006). Design research from a technology perspective. In J. V. D. Akker, K. Gravemeijer, S. McKenney \& N. Nieveen (Eds.), Educational design research (pp. 52-66). New York: Routledge.

Reimann, P. (2016). Connecting learning analytics with learning research: the role of design-based research. Learning: Research \& Practice, 2(2), 130-142.

Rogers, T. (2015). Critical realism and learning analytics research: epistemological implications of an ontological foundation. In Proceedings of the Fifth International Conference on Learning Analytics And Knowledge (pp. 223-230). ACM.

Rowntree D. (1987). Assessing students: How shall we know them? London: Kogan Page.

Siemens, G. (2013). Learning analytics: The emergence of a discipline. American Behavioral Scientist, 57 (10): 1380-1400.

Siemens, G., \& Baker, R. S. (2012). Learning analytics and educational data mining: Towards communication and collaboration. In S. Buckingham Shum, D. Gašević, \& R. Ferguson (Eds.), Proceedings of the 2nd International Conference on Learning Analytics and Knowledge (pp. 252254). New York, NY: ACM.

Smoliar, S. W. (1987) Book review: "Induction: Processes of inference, learning, and discovery". IEEE Expert, 2 (3). 92-93.

Soderstrom, N. C., \& Bjork, R. A. (2015). Learning versus performance: An integrative review. Perspectives on Psychological Science, 10(2), 176199.

Strickland, J. S. (2014). Predictive modelling and analytics. Colorado: Lulu Press.

Wise, A. F. \& Shaffer, D. W. (2015). Why theory matters more than ever in the age of big data. Journal of Learning Analytics, 2, 5-13.

Yarkoni, T. (2012). Psychoinformatics: New horizons at the interface of the psychological and computing sciences. Current Directions in Psychological Science, 21(6), 391-397. doi: 10.1177/0963721412457362

Zliobaite, I., Bifet, A., Gaber, M., Gabrys, B., Gama, J., Minku, L., \& Musial, K. (2012). Next challenges for adaptive learning systems. ACM SIGKDD Explorations Newsletter, 14(1), 48-55. 


\section{University Library}

\section{- M M N E R VA A gateway to Melbourne's research publications}

Minerva Access is the Institutional Repository of The University of Melbourne

Author/s:

Lodge, JM;Alhadad, SSJ;Lewis, MJ;Gasevic, D

Title:

Inferring Learning from Big Data: The Importance of a Transdisciplinary and Multidimensional Approach

Date:

2017-10-01

Citation:

Lodge, J. M., Alhadad, S. S. J., Lewis, M. J. \& Gasevic, D. (2017). Inferring Learning from Big Data: The Importance of a Transdisciplinary and Multidimensional Approach. TECHNOLOGY KNOWLEDGE AND LEARNING, 22 (3), pp.385-400. https://doi.org/10.1007/ s10758-017-9330-3.

Persistent Link:

http://hdl.handle.net/11343/282965 